\section{Avaliação da qualidade das informações relativas aos óbitos em menores de um ano em Blumenau,1998}

\author{
Quality assessment of death related data of \\ under one year old infants in Blumenau, \\ 1998
}

Ernani Tiaraju de Santa Helena 1

Maurício Bittencourt Rosa 2

1,2 Universidade Regional de Blumenau. Departamento de Medicina. Rua Antonio da Veiga, 140. Blumenau, SC, Brasil. CEP: 89.010-97

\begin{abstract}
Objectives: to quantify inaccurate and missing information and to assess the quality of data related to infant deaths in Blumenau during the year of 1998.

Methods: information was obtained on 125 deaths entered in the civil registration records and of 12 other deaths from hospitals in Blumenau. A new Death Certificate was filled (New DC) for comparison with each original Death Certificate (Original DC) based on hospital records information. New coefficients of child mortality and underlying causes were compared with the original information and the consistency between variables was determined.

Results: an inaccuracy of $8,8 \%$ of the death records were demonstrated with the following outcomes: official coefficients are Child Mortality Coefficient 16,7; Perinatal Mortality Coefficient 21,0, Neonatal Mortality Coefficient 3,9, and the new ones depicted, 18,0, 23,1, 13,8 and 4,1 respectively. Social and demographic variables of fetal and non-fetal deaths are poorly reported not only in the Death Certificates but in hospital records as well. In general the Original Death Certificates for fetal deaths are worse than those of non-fetal deaths. The Kappa index between Original Death Certificates and New Death Certificates for perinatal causes and congenital malformations was of 0,73 and 0,80 respectively.

Conclusions: notwithstanding the deficiency of information and unsatisfactory quality of the social and demographic data of infant deaths, the causes could be used for health planning considering their consistency.
\end{abstract}

Key words Child mortality, information systems, basic causes of death, death records.

\section{Resumo}

Objetivos: quantificar o sub-registro e avaliar a qualidade das informações dos óbitos infantis em Blumenau, durante o ano de 1998.

Métodos: foram obtidas informações de 125 óbitos registrados nos cartórios de registro civil e de outros 12 óbitos junto aos hospitais de Blumenau. Preencheu-se uma Declaração de Óbito Nova (DO Nova) para comparação com cada Declaração de Óbito Original (DO Original), com base nas informações dos prontuários hospitalares. Foram comparados os novos coeficientes de mortalidade infantil e sub-componentes com os originais e determinada a concordância entre variáveis.

Resultados: observou-se um sub-registro de $8,8 \%$ de óbitos, verificando-se que: os coeficientes oficiais são Coeficiente de Mortalidade Infantil 16,7; Coeficiente de Mortalidade Perinatal 21,0; Coeficiente de Mortalidade Neonatal 12,8; Coeficiente de Mortalidade Pós-neonatal 3,9; e os novos são 18,0; 23,0; 13,8; e 4,1 respectivamente. As variáveis sócio-demográficas dos óbitos fetais e não fetais são mal preenchidas tanto na DO Original como nos prontuários médicos. No geral, as DO Originais de óbitos fetais são pior preenchidas que as de óbitos não fetais. O indice Kappa entre as DOs Originais e Novas para causas perinatais e malformações congênitas foi de 0,73 e 0,80, respectivamente.

Conclusões: apesar do sub-registro e da insatisfatória qualidade das informações sócio-demográficas dos óbitos infantis, as causas podem ser utilizadas para planejamento em saúde em vista da boa concordância observada.

Palavras-chave Mortalidade infantil, Sistemas de informação, Causa básica da morte, Registros de mortalidade 


\section{Introdução}

Os indicadores de saúde relacionados à mortalidade são, em grande parte, construídos com dados existentes nas declarações de óbito (DO). Esses dados podem apresentar inconsistências prejudicando a confiabilidade dessas informações.

O coeficiente de mortalidade infantil (CMI) expressa o risco de um nascido vivo morrer antes de completar um ano de idade. ${ }^{1}$ A morte de menores de um ano está relacionada diretamente com as condições de saneamento, habitação, educação, nutrição, assistência pré-natal e ao parto, ou seja, as condições socioeconômicas da população. ${ }^{2}$

A distorção quantitativa (abrangência total dos casos), pode gerar indicadores e ações de saúde equivocadas. ${ }^{3} \mathrm{O}$ registro adequado dos óbitos é fundamental para o setor saúde, tanto para o planejamento, quanto em estudos epidemiológicos.

Do ponto de vista qualitativo, estudos nacionais ressaltam a importância do preenchimento adequado da DO, tanto nos campos de identificação como, principalmente, no da causa básica da morte. ${ }^{4-7}$ Porém, o que se verifica é que os médicos continuam a não preencher corretamente as DOs, talvez por não terem sido devidamente ensinados nas escolas médicas, ou por que, para a maioria deles, o atestado possui apenas finalidade legal. ${ }^{8}$ Apesar da importância do tema, não existem estudos relativos a essa pro-blemática em Santa Catarina.

O objetivo do presente trabalho é quantificar o sub-registro e avaliar a qualidade das informações relativas aos óbitos em menores de um ano em Blumenau, no ano de 1998.

\section{Métodos}

Trata-se de um estudo descritivo exploratório, retrospectivo, observacional com unidade de estudo individual.

Foram investigados 137 óbitos em menores de um ano residentes em Blumenau, SC, Brasil, ocorridos de um de janeiro de 1998 a 31 de dezembro de 1998. Utilizou-se como fonte de informação as Declarações de Óbito (DO Original) processadas pelo Sistema de Informação de Mortalidade (SIM) da Secretaria Municipal de Saúde de Blumenau (SEMUS). Os óbitos foram separados em dois grupos: 125 óbitos (48 fetais e 77 não fetais, dos quais 49 até sete dias) cujas DOs foram registradas nos cartórios de registro civil de Blumenau e 12 óbitos (seis fetais e seis não fetais, dos quais três até sete dias) com DOs obtidas junto aos hospitais públicos e privados da cidade (não registradas).

A partir desses óbitos identificados, foi preenchida uma Declaração de Óbito Nova (DO Nova) para cada óbito registrado em cartório (DO Original), com base nas informações obtidas através dos prontuários médicos hospitalares. Esta DO Nova foi preenchida por um acadêmico de Medicina para todos os campos constantes nas unidades II (identificação do falecido), III (óbito fetal ou menor de um ano), IV (atestado médico), VI (causas externas) da Declaração de Óbito, exceto no campo relativo à causa básica da morte que foi preenchido por um pediatra com especialização em saúde pública. Ambos (acadêmico e pediatra) não tinham conhecimento prévio do conteúdo das DO Originais.

As variáveis de estudo foram divididas em: sócio-demográficas: sexo, idade, ocupação habitual pai, grau de instrução do pai, idade da mãe, ocupação habitual mãe, grau de instrução da mãe; dados relacionados à gestação e o parto: número de filhos tidos, duração da gestação, gravidez, parto, peso ao nascer; assistência médica: recebeu assistência médica durante a doença que ocasionou a morte?, o médico que assina atendeu ao falecimento?, o diagnóstico foi confirmado?; causa básica do óbito.

Os dados da DO nova foram codificados utilizando-se a mesma metodologia utilizada pelo Sistema de Informação de Mortalidade. A codificação das causas de óbito foi feita com base na Classificação Internacional de Doenças, ${ }^{9}$ sendo realizada por técnico treinado pelo Centro Brasileiro de Classificação de Doenças. A causa básica foi determinada utilizando-se o aplicativo Sistema de Causa Básica (SCB) do Sistema de Informação de Mortalidade (SIM), do Ministério da Saúde. As causas dos óbitos foram apresentadas isoladas e em grupos de causas, conforme a Classificação Brasileira de Mortalidade. ${ }^{9}$

Os dados foram digitados em banco de dados eletrônico. Foram calculados os coeficientes de mortalidade infantil, neonatal (CMN), pós-neonatal (CMPo) e perinatal (CMPe), tendo-se utilizado no numerador os óbitos registrados em cartório. Novos coeficientes foram calculados utilizando-se todos os óbitos obtidos (registrados + não registrados). O denominador (número de nascidos vivos no ano de 1998) foi de 4.610 nascidos vivos, informação obtida junto ao Sistema de Nascidos Vivos (SINASC) de Blumenau. Para se estimar o sub-registro dividiu-se o número de óbitos não registrados pelo total de óbitos (registrados + não registrados) multiplicado por cem.

Utilizou-se de estatística descritiva com freqüência simples e número absoluto (devido ao pequeno número de óbitos) e apresentação tabular. Para se 
analisar a concordância entre os grupos de causas (causas perinatais, malformações congênitas e outras) obtidos a partir da DO Original e da DO Nova, empregou-se o índice Kappa, que leva em consideração, além da concordância observada $(\mathrm{CO})$, aquela que seria esperada pelo mero acaso. Considera-se um índice maior ou igual a 0,75 como elevada concordância, abaixo de 0,40 como baixa concordância e entre esses valores, como boa concordância. 10

\section{Resultados}

Na revisão dos prontuários hospitalares, foram encontrados 12 óbitos não registrados em Cartório, caracterizando um sub-registro de $8,8 \%$. Desses, seis eram de óbitos fetais (sub-registro de $11,1 \%$ ) e seis de não fetais (sub-registro de 7,3\%). Em relação aos não fetais, três foram a óbito até sete dias, ou seja, nove óbitos perinatais não foram registrados, isto é, um sub-registro de $8,5 \%$. A Tabela 1 apresenta o coeficiente de mortalidade infantil e sub-componentes oficiais e os novos.

\begin{tabular}{|c|c|c|c|c|c|}
\hline & $\mathrm{n}$ & Coeficiente & $\mathrm{n}$ & Coeficiente & Novos/Oficiais \\
\hline Mortalidade perinatal & 106 & 23,0 & 97 & 21,0 & 1,093 \\
\hline Mortalidade neonatal & 64 & 13,8 & 59 & 12,8 & 1,085 \\
\hline Mortalidade pós-neonatal & 19 & 4,1 & 18 & 3,9 & 1,056 \\
\hline
\end{tabular}

Para avaliação da qualidade das informações apresentadas a seguir, foram estudados somente os 87 óbitos em menores de um ano ocorridos no município de Blumenau em 1998, que tiveram seus prontuários hospitalares localizados.

Com relação à caracterização do tipo de óbito (fetal ou não fetal), na DO Original foram encontrados $36(41,4 \%)$ de óbitos fetais e $51(58,6 \%)$ de óbitos não fetais, enquanto na DO Nova foram encontrados $37(42,5 \%)$ de óbitos fetais e $50(57,5 \%)$ de óbitos não fetais.

As variáveis de estudo foram inicialmente estudadas quanto ao seu preenchimento (presença ou ausência de informação). A Tabela 2 apresenta as informações ignoradas e em branco das DOs Originais e das DOs Novas, segundo o tipo de óbito. As variáveis referentes às condições sócio-demográficas são mal preenchidas tanto na DO Original quanto nos prontuários hospitalares, sendo o pior campo aquele referente à instrução do pai, com $(86,1 \%$ e $97,3 \%$, respectivamente). De modo geral, as DOs Originais relativas aos óbitos fetais têm mais informações omissas do que as DOs Originais dos óbitos não fetais, tanto no que se refere às variáveis relacionadas à gestação e ao parto e às assistenciais.

A Tabela 3 mostra dados de identificação dos casos de morte não fetal em menores de um ano. $\mathrm{O}$ sexo masculino é predominante com (70\%). O maior número de óbitos ocorreu no período neonatal precoce $(72 \%)$ e o menor número no período neonatal tardio (12\%). 
Tabela 2

Distribuição das informações ignoradas e em branco das DOs Original e Nova, segundo tipo de óbito e variáveis de estudo. Blumenau, SC, Brasil, 1998.

\begin{tabular}{|c|c|c|c|c|c|c|c|c|}
\hline \multirow{3}{*}{ Tipos de variáveis } & \multicolumn{4}{|c|}{ Fetal } & \multicolumn{4}{|c|}{ Não fetal } \\
\hline & \multicolumn{2}{|c|}{ DO Original } & \multicolumn{2}{|c|}{ DO Nova } & \multicolumn{2}{|c|}{ DO Original } & \multicolumn{2}{|c|}{ DO Nova } \\
\hline & $n$ & $\%$ & $\mathrm{n}$ & $\%$ & $\mathrm{n}$ & $\%$ & $\mathrm{n}$ & $\%$ \\
\hline \multicolumn{9}{|l|}{ Sócio-demográficas } \\
\hline Sexo & 1 & 2,8 & 6 & 16,2 & 0 & 0,0 & 0 & 0,0 \\
\hline Ocupação do pai & 17 & 47,2 & 24 & 66,0 & 28 & 54,9 & 42 & 84,0 \\
\hline Instrução do pai & 31 & 86,1 & 36 & 97,3 & 33 & 64,7 & 49 & 98,0 \\
\hline Ocupação da mãe & 12 & 33,3 & 5 & 13,5 & 24 & 47,1 & 33 & 66,0 \\
\hline Idade da mãe & 10 & 27,7 & 2 & 5,4 & 9 & 17,6 & 30 & 60,0 \\
\hline Instrução da mãe & 26 & 72,2 & 29 & 78,4 & 12 & 23,5 & 42 & 84,0 \\
\hline \multicolumn{9}{|l|}{ Gestação e parto } \\
\hline Filhos vivos & 24 & 66,6 & 5 & 13,5 & 31 & 60,7 & 22 & 44,0 \\
\hline Filhos mortos & 32 & 88,8 & 5 & 13,5 & 49 & 96,1 & 21 & 42,0 \\
\hline Semanas de gestação & 8 & 22,2 & 8 & 21,6 & 8 & 15,6 & 22 & 44,0 \\
\hline Tipo de gravidez & 13 & 36,1 & 1 & 2,7 & 6 & 11,7 & 16 & 32,0 \\
\hline Tipo de parto & 13 & 36,1 & 1 & 2,7 & 8 & 15,6 & 15 & 30,0 \\
\hline Peso ao nascer & 15 & 41,6 & 15 & 40,5 & 7 & 13,5 & 12 & 24,0 \\
\hline \multicolumn{9}{|l|}{ Assistenciais } \\
\hline Recebeu assistência & 21 & 58,3 & 0 & 0,0 & 11 & 21,6 & 0 & 0,0 \\
\hline Exame complementar & 12 & 33,3 & 0 & 0,0 & 12 & 23,5 & 0 & 0,0 \\
\hline Cirurgia & 17 & 47,2 & 0 & 0,0 & 18 & 35,3 & 0 & 0,0 \\
\hline Necropsia & 16 & 44,4 & 0 & 0,0 & 17 & 33,3 & 0 & 0,0 \\
\hline
\end{tabular}

DO = Declaração de óbito

Fonte: Blumenau. Secretaria de Saúde. SIM (Sistema de Informação de Mortalidade). Prontuários hospitalares; 1998.

Tabela 3

Distribuição das informações das DOs Original e Nova relativas a identificação do falecido nos casos de morte não fetal em menores de um ano. Blumenau, SC, Brasil, 1998.

\begin{tabular}{|c|c|c|c|c|c|}
\hline \multirow{2}{*}{ Identificação } & & \multicolumn{2}{|c|}{ DO Original } & \multicolumn{2}{|c|}{ DO Nova } \\
\hline & & $\mathrm{n}$ & $\%$ & $\mathrm{n}$ & $\%$ \\
\hline \multirow[t]{2}{*}{ Sexo } & Masculino & 37 & 72,5 & 35 & 70,0 \\
\hline & Feminino & 14 & 27,5 & 15 & 30,0 \\
\hline \multirow[t]{3}{*}{ Faixa etária } & Até sete dias & 37 & 72,6 & 36 & 72,0 \\
\hline & $8-27$ dias & 6 & 11,7 & 6 & 12,0 \\
\hline & 28 dias - 11 meses e 29 dias & 8 & 15,7 & 8 & 16,0 \\
\hline
\end{tabular}

DO = Declaração de óbito

Fonte: Blumenau. Secretaria de Saúde. SIM (Sistema de Informação de Mortalidade). Prontuários hospitalares; 1998. 
A Tabela 4 mostra as informações sócio-demográficas dos óbitos infantis não fetais. Em relação à ocupação do pai houve uma predominância de agricultores e industriários na DO Original (60\%) e na DO Nova $(62,5 \%)$. Para os pais sem nenhuma instrução, a maioria ocorreu na DO Original $(73,3 \%)$, enquanto que na DO Nova apenas uma informação foi encontrada nos prontuários. Mães técnicas, gerentes, e com nível superior foram a maioria na DO Original $(65,51 \%)$. Entretanto, mães funcionárias públicas, trabalhadoras em serviços e no comércio em geral predominaram na DO Nova $(47,05 \%)$.

Houve uma boa concordância no que tange à idade e ao grau de instrução da mãe. A idade mais freqüente da mãe foi de 20 - 29 anos na DO Original $(60,4 \%)$ e na DO Nova (65\%); o grau de instrução mais frequiente foi o primeiro grau na DO Original $(70,5 \%)$ e na DO Nova $(75 \%)$.

No que se refere a informações gestacionais e do parto, observou-se que a duração predominante da gestação foi de 28 a 36 semanas tanto para as DOs Original $(54,5 \%)$ quanto para as DO Nova $(67,5 \%)$. $\mathrm{O}$ parto operatório e a gravidez única perfizeram a maioria em ambas. Houve discordância entre o peso das crianças, pois na DO Original o peso mais freqüente foi o de 1.000 a $2.499 \mathrm{~g}(43,18 \%)$; já na DO Nova foi de 2.500 e mais (42\%) (Tabela 5).

\section{Tabela 4}

Distribuição das informações das DOs Original e Nova relativas a informações sócio-demográficas dos óbitos infantis não fetais. Blumenau, SC, Brasil, 1998.

\begin{tabular}{|c|c|c|c|c|c|}
\hline \multirow{2}{*}{\multicolumn{2}{|c|}{ Informações sócio-demográficas }} & \multicolumn{2}{|c|}{ DO Original } & \multicolumn{2}{|c|}{ DO Nova } \\
\hline & & \multirow{2}{*}{$\begin{array}{r}n \\
-\end{array}$} & \multirow{2}{*}{$\begin{array}{r}\% \\
\\
\end{array}$} & \multirow{2}{*}{$\frac{n}{2}$} & \multirow{2}{*}{$\begin{array}{c}\% \\
25,0\end{array}$} \\
\hline Ocupação do pai & Técnicos, gerentes, nível superior & & & & \\
\hline & Serviços, comércio & 10 & 40,0 & 1 & 12,5 \\
\hline & Agricultores e industriários & 15 & 60,0 & 5 & 62,5 \\
\hline \multirow[t]{4}{*}{ Instrução do pai } & Nenhuma & 22 & 73,3 & - & - \\
\hline & Primeiro grau & 1 & 3,3 & - & - \\
\hline & Segundo grau & 6 & 20,0 & 1 & 100,0 \\
\hline & Superior & 1 & 3,3 & - & - \\
\hline \multirow[t]{3}{*}{ Ocupação da mãe } & Técnicos, gerentes, nível superior & 19 & 65,5 & 4 & 23,5 \\
\hline & Funcionários públicos, serviços, comércio & 5 & 17,2 & 8 & 47,1 \\
\hline & Agricultores e industriários & 5 & 17,2 & 5 & 29,4 \\
\hline \multirow[t]{4}{*}{ Idade da mãe } & $10-19$ & 9 & 20,9 & 4 & 20,0 \\
\hline & $20-29$ & 26 & 60,4 & 13 & 65,0 \\
\hline & $30-39$ & 8 & 18,6 & 3 & 15,0 \\
\hline & 40 e mais & 1 & 2,3 & - & - \\
\hline \multirow[t]{4}{*}{ Instrução da mãe } & Nenhuma & 5 & 11,4 & - & - \\
\hline & Primeiro grau & 31 & 70,5 & 6 & 75,0 \\
\hline & Segundo grau & 6 & 13,6 & 2 & 25,0 \\
\hline & Superior & 2 & 4,5 & - & - \\
\hline
\end{tabular}

$\mathrm{DO}=$ Declaração de óbito

Fonte: Blumenau. Secretaria de Saúde. SIM (Sistema de Informação de Mortalidade). Prontuários hospitalares; 1998. 
Tabela 5

Distribuição das informações das DOs Original e Nova relativas a gestação e ao parto em menor de um ano nos caso de morte não fetal infantil. Blumenau, SC, Brasil, 1998.

\begin{tabular}{|c|c|c|c|c|c|}
\hline \multirow{2}{*}{\multicolumn{2}{|c|}{ Informações relativas a gestação e parto }} & \multicolumn{2}{|c|}{ DO Original } & \multicolumn{2}{|c|}{ DO Nova } \\
\hline & & $\mathrm{n}$ & $\%$ & $\mathrm{n}$ & $\%$ \\
\hline \multirow[t]{5}{*}{ Número de nascidos vivos } & 1 & 10 & 50,0 & 11 & 39,2 \\
\hline & 2 & 7 & 35,0 & 11 & 39,2 \\
\hline & 3 & 1 & 5,0 & 5 & 17,8 \\
\hline & 4 & 1 & 5,0 & 1 & 3,5 \\
\hline & 5 & 1 & 5,0 & - & - \\
\hline \multirow[t]{5}{*}{ Número de nascidos mortos } & 0 & - & - & 18 & 62,1 \\
\hline & 1 & 2 & 100,0 & 7 & 24,1 \\
\hline & 2 & - & - & 1 & 3,4 \\
\hline & 3 & - & - & 2 & 6,8 \\
\hline & 9 & - & - & 1 & 3,4 \\
\hline \multirow[t]{3}{*}{ Gravidez } & Única & 39 & 86,7 & 28 & 77,7 \\
\hline & Dupla & 6 & 13,3 & 8 & 22,2 \\
\hline & Tríplice & - & - & - & - \\
\hline \multirow[t]{3}{*}{ Parto } & Espontâneo & 16 & 37,2 & 14 & 40,0 \\
\hline & Operatório & 27 & 62,7 & 20 & 57,0 \\
\hline & Fórceps & - & - & 1 & 2,8 \\
\hline \multirow[t]{5}{*}{ Duração da gestação (semanas) } & 0 a 21 & 1 & 2,3 & - & - \\
\hline & 22 a 27 & 8 & 18,2 & 6 & 21,4 \\
\hline & 28 a 36 & 24 & 54,5 & 19 & 67,8 \\
\hline & 37 a 41 & 11 & 25,0 & 3 & 10,7 \\
\hline & 42 e mais & - & - & - & - \\
\hline \multirow[t]{3}{*}{ Peso ao nascer $(\mathrm{g})$} & Até 999 & 10 & 22,7 & 11 & 22,0 \\
\hline & 1.000 a 2.499 & 19 & 43,1 & 18 & 36,0 \\
\hline & 2.500 e mais & 15 & 34,1 & 21 & 42,0 \\
\hline
\end{tabular}

DO = Declaração de óbito

Fonte: Blumenau. Secretaria de Saúde. SIM Sistema de Informação de Mortalidade). Prontuários hospitalares; 1998.

Todas as crianças menores de um ano que foram a óbito receberam assistência médica, sendo que os exames complementares foram os que mais confirmaram diagnóstico: na DO Original 79,4\% e 78,0\% na DO Nova; a necrópsia foi a que menos confirmou: $2,9 \%$ na DO Original e $0 \%$ na DO Nova.

Os dados da Tabela 6 mostram que o grau de concordância é bom entre as DOs Original e Nova em relação à causa básica da morte, com destaque para a síndrome de angústia respiratória do recém nascido. A concordância observada para causa básica do óbito entre as DOs Original e DO Nova para o grupo das afecções originadas no período perinatal foi de 0,91 com um índice Kappa de 0,73 (p < 0,001) enquanto para o grupo das malformações congênitas a concordância observada foi de 0,94 e o índice Kappa de $0,80(\mathrm{p}<0,001)$. 
Distribuição das informações das DOs Original e Nova relativas a gestação e ao parto em menor de um ano nos caso de morte não fetal infantil. Blumenau, SC, Brasil, 1998.

\begin{tabular}{|c|c|c|c|c|c|c|}
\hline \multirow{2}{*}{ Informações relativas a gestação e parto } & \multicolumn{2}{|c|}{ DO Original } & \multicolumn{2}{|c|}{ DO Nova } & \multicolumn{2}{|c|}{ Concordância } \\
\hline & $\mathrm{n}$ & $\%$ & $\mathrm{n}$ & $\%$ & Observação & Kappa \\
\hline Outras Causas & 3 & 6,0 & 3 & 6,0 & & \\
\hline Encefalopatia não especificada & 1 & 2,0 & 1 & 2,0 & & \\
\hline Doenças infecciosas e parasitárias & 1 & 2,0 & 0 & 0,0 & & \\
\hline Doenças respiratórias & 1 & 2,0 & 2 & 4,0 & & \\
\hline Afecções do período perinatal & 33 & 66,0 & 32 & 64,0 & 0,91 & 0,73 \\
\hline $\begin{array}{l}\text { Síndrome da angústia respiratória do recém- } \\
\text { nascido }\end{array}$ & 18 & 36,0 & 20 & 40,0 & & \\
\hline Sepsis & 2 & 4,0 & 4 & 8,0 & & \\
\hline Outras afeccões respiratórias & 7 & 14,0 & 6 & 12,0 & & \\
\hline Outros & 6 & 12,0 & 0 & 0,0 & & \\
\hline Morte fetal não especificada & 0 & 0,0 & 2 & 4,0 & & \\
\hline Malformações congênitas & 14 & 28,0 & 15 & 30,0 & 0,94 & 0,80 \\
\hline Anencefalia & 3 & 6,0 & 3 & 6,0 & & \\
\hline Mal formação do coração & 5 & 10,0 & 5 & 10,0 & & \\
\hline Hérnia diafragmática congênita & 3 & 6,0 & 4 & 8,0 & & \\
\hline Outras malformações e NCOP & 3 & 6,0 & 3 & 6,0 & & \\
\hline
\end{tabular}

DO = Declaração de óbito

Fonte: Blumenau. Secretaria de Saúde. SIM Sistema de Informação de Mortalidade). Prontuários hospitalares; 1998.

\section{Discussão}

Merece destaque inicial o fato que algumas DOs não puderam ser refeitas devido ao mau preenchimento do prontuário médico, em especial no que se refere a óbitos fetais. Tal situação poderia ser parcialmente contornada caso fosse realizada necropsia dos óbitos, o que não foi possível pois não existe um Serviço de Verificação de Óbitos (SVO) em Blumenau.

O sub-registro de óbitos pode afetar os indicadores de mortalidade infantil e seus componentes, promovendo uma queda artificial dos coeficientes e levando à falsa impressão de que as condições de saúde são satisfatórias. Autores nacionais detectaram um sub-registro em Pelotas de $45 \%$ e em Porto Alegre de $3 \%$ dos óbitos. 4 Os resultados obtidos sugerem que o sub-registro acontece em Blumenau e que pode ser até maior que o observado, pois não foi investigada no estudo a possibilidade de enterros clandestinos. 1
O maior sub-registro em Blumenau foi dos óbitos fetais, afetando o coeficiente de mortalidade perinatal. Barros et al. 11 obtiveram em seu estudo um sub-registro de óbito perinatais de $36 \%$. Outro estudo realizado em Pelotas aponta uma redução no sub-registro de óbitos perinatais de 42,1\% em 1982 para $6,8 \%$ em 1993, esse último próximo ao índice encontrado em nosso estudo. ${ }^{12}$ Tal achado tem importantes implicações, pois esse coeficiente relaciona-se com a assistência médica pré-natal e o parto, além de apresentar forte associação com o baixo nível socioeconômico dos pais e idade materna maior que 35 anos. ${ }^{13}$

Mesmo tendo em conta o sub-registro encontrado em nosso estudo, os coeficientes ajustados se mostram com valores melhores que os obtidos em cidades de várias regiões brasileiras, tais como Pelotas, RS no ano de 1993 (CMI = 21,1/1.000, $\mathrm{CMN}=14,3 / 1.000$ e CMP $=6,9 / 1.000),{ }^{13}$ Rio de Janeiro, RJ em $1995(\mathrm{CMI}=21,8 / 1.000, \mathrm{CMN}=$ 14,4/1.000 e CMP = 7,3/1.000) ${ }^{14}$ e Salvador, BA em 
$1997(\mathrm{CMI}=27,9 / 1.000, \mathrm{CMN}=18,8 / 1.000$ e $\mathrm{CMP}$ =9,1/1.000). 15

Com relação à qualidade da informação, as informações relativas aos óbitos fetais foram melhor preenchidas no que diz respeito às variáveis assistenciais, em comparação com as dos óbitos não fetais. Em contrapartida, as variáveis relacionadas à gestação e ao parto dos óbitos não fetais apresentaram menos informações ignoradas do que a dos óbitos fetais. Isto pode ser explicado porque os prontuários mãe/filho são mais completos.

As condições sócio-demográficas, tais como escolaridade e ocupação dos pais, têm sido apontadas como fatores preditivos de óbitos infantis e seus subcomponentes. 12,16 Essas foram mal preenchidas tanto nas DOs Originais, como nos prontuários hospitalares. Os campos relativos a essas variáveis são, em geral, preenchidos pelos setores administrativos dos hospitais e merecem um tratamento diferenciado. Os campos relativos à gestação e ao parto são preenchidos pelo médico assistente, e sua má qualidade parece indicar mais uma falta de comprometimento do profissional de saúde no registro dessas informações, 17 do que um desconhecimento do conteúdo que tinha de ser registrado nos prontuários. Este conjunto de variáveis, em especial o peso ao nascer e a idade gestacional, representam importantes fatores preditivos de óbito tendo-se obtido neste estudo menor proporção de ignorados e em branco do que outros estudos nacionais, que têm oscilado entre 27 a $82 \% .6,17,18$

Uma estratégia empregada pela Secretaria $\mathrm{Mu}$ nicipal de Saúde (que processa as informações dos

\section{Agradecimentos}

À Universidade Regional de Blumenau que financiou essa pesquisa por meio do Programa de Incentivo à Pesquisa (PIPe).

\section{Referências}

1. Laurenti R, Mello Jorge MHP, Lebrão ML, Gotlieb SLD. Estatísticas de saúde. São Paulo: EPU; 1985.

2. Monteiro CA. Contribuição para o estudo do significado da evolução do coeficiente de mortalidade infantil no município de São Paulo, SP (Brasil), nas três últimas décadas (1950-1980). Rev Saúde Pública 1982; 16: 7-18.

3. Mello Jorge MHP, Gotlieb SLD, Soboll MLMS, Almeida óbitos) para melhorar a qualidade da informação tem sido de utilizar as informações disponíveis nos Cartórios ou do SINASC para completar os campos não preenchidos nas DOs Originais, o que pode justificar a melhor qualidade em relação as DOs Novas preenchidas com base nos prontuários médicos. Este tipo de estratégia têm também sido usada por outros serviços com resultados satisfatórios para o aprimoramento das informações. 19 Outros autores sugerem o uso de técnica de linkage entre os bancos de dados de mortalidade (SIM) e de nascidos vivos (SINASC), como forma de otimizar a obtenção de informações não preenchidas nas Declarações de Óbito. 20 Contudo, esse método não permite a correção das informações relativas aos óbitos fetais, que não constam no SINASC; mas podem ser feitas através de visitas domiciliares de rotina.

No que tange à causa básica, observou-se uma elevada concordância entre a DO Original e a DO Nova, considerada como padrão ouro, o que sugere que este campo da DO Original pode ser utilizado em estudos epidemiológicos e como subsídio para planejamento de ações. Estudos nacionais são controversos a esse respeito, pois enquanto no Rio de Janeiro observou-se que apenas $52,3 \%$ das declarações mantiveram a mesma causa básica, em Belo Horizonte obteve-se índice Kappa de 0,77 para causas perinatais e 0,72 para malformações congênitas. 21,22 Outro estudo nacional também obteve concordância elevada para malformações congênitas (Kappa $=0,85)$ e boa concordância para imaturidade $($ Kappa $=0,72) \cdot 18$

MF, Oliveira MRD. Avaliação do sistema de informação sobre nascidos vivos e o uso de seus dados em epidemiologia e estatísticas de saúde. Rev Saúde Publica 1993; 27: 1-46.

4. Nobre LC, Victora CG, Barros FC, Lombardi C, Teixeira AMB, Fuchs SC. Avaliação da qualidade da informação sobre a causa básica de óbitos infantis no Rio Grande do 
Sul (Brasil). Rev Saúde Pública 1989; 23: 207-13.

5. Carvalho ML, Silver LD. Confiabilidade da declaração da causa básica de óbitos neonatais: implicações para o estudo da mortalidade previnível. Rev Saúde Pública 1995; 29: 342-8.

6. Heckmann IC, Canani LH, Sant'Anna UL, Bordin R. Análise do preenchimento de declarações de óbito em localidade do estado do Rio Grande do Sul (Brasil), 1987. Rev Saúde Pública 1989; 23: 292-7.

7. Strozzi GM, Strozzi JB, Souza ML, Schutel MD. Estudo de causa básica de óbitos de menores de quinze anos, ocorridos em Hospital de Florianópolis, SC (Brasil), em 1982. Rev Saúde Pública 1985; 19: 123 -32.

8. Laurenti, R. Mortalidade infantil nos Estados Unidos, Suécia e Estado de São Paulo. Rev Saúde Pública 1987; 21 : 268-73.

9. OMS (Organização Mundial da Saúde). Classificação internacional de doenças. 10. rev. São Paulo: Centro Brasileiro de Classificação de Doenças; 1985.

10. Fleiss, JL. Statistical methods for rates and proportions. New York: Wiley; 1981.

11. Barros FC, Victora CG, Teixeira AMB, Puerto Filho M. Mortalidade perinatal e infantil em Pelotas, Rio Grande do Sul: nossas estatísticas são confiáveis? Cad Saúde Pública 1985; 1: 348-58.

12. Menezes AM, Barros FC, Victora CG, Alves C, Rocha C, Albernaz E, Menezes FS, Jannke HA. Mortalidade perinatal em duas coortes de base populacional no sul do Brasil: tendências e diferenciais. Cad Saúde Pública 1996; 12 (Supl 1): 33-41.

13. Menezes AMB, Barros FC, Victora CG, Tomasi E, Halpern R, Oliveira ALB. Fatores de risco para mortalidade perinatal em Pelotas, RS, 1993. Rev Saúde Publica 1998; 32 : 209-16.

14. Campos TP, Carvalho MS, Barcellos CC. Mortalidade in- fantil no Rio de Janeiro, Brasil: áreas de risco e trajetória dos pacientes até os serviços de saúde. Rev Panam Salud Publica 2000; 8: 164-71.

15. Costa MCN, Azi PA, Paim JS, Silva LMV. Mortalidade infantil e condições de vida: a reprodução das desigualdades sociais em saúde na década de 90. Cad Saúde Pública 2001; 17: 555-67.

16. França E, Souza JM, Guimaräes MDC, Goulart EMA, Colosimo E, Antunes CMF. Associação entre fatores sócio-econômicos e mortalidade infantil por diarréia, pneumonia e desnutrição em região metropolitana do Sudeste do Brasil: um estudo caso-controle. Cad Saúde Pública 2001; 17: 1437-47.

17. Pereira MG, Castro ES. Avaliação do preenchimento de declarações de óbitos. Rev Saúde Publica 1981; 15: 14-9.

18. Gomes JO, Santo AH. Mortalidade infantil em município da região Centro-Oeste Paulista, Brasil, 1990 a 1992. Rev Saúde Pública 1997; 31: 330-41.

19. Noronha CP, Silva RI, Theme Filha MM. Concordância de dados das declarações de óbito e de nascidos vivos para a mortalidade neonatal no município do Rio de Janeiro. Inf Epidemiol SUS 1997; 8: 57-65.

20. Almeida MF, Mello Jorge MHP. O uso da técnica de "linkage" de sistemas de informação em estudos de coorte sobre mortalidade neonatal. Rev Saúde Pública 1996; 30: 141-7.

21. Niobey FML, Cascão AM, Duchiade MP, Sabroza PC. Qualidade do preenchimento de atestados de óbitos de menores de um ano na região metropolitana do Rio de Janeiro. Rev Saúde Pública 1990; 24: 311-8.

22. Mendonça EF, Goulart EMA, Machado JAD. Confiabillidade da declaração de causa básica de mortes infantis em região metropolitana do sudeste do Brasil. Rev Saúde Pública 1994; 5: 385-91.

Recebido em 6 de março de 2002

Versão final reapresentada em 18 junho de 2002

Aprovado em 15 de outubro de 2002 